\title{
LA SALUD MATERNO-INFANTIL DURANTE EL FRANQUISMO: NOTAS BIBLIOMÉTRICAS SOBRE EL PROGRAMA «AL SERVICIO DE ESPAÑA Y DEL NIÑO ESPAÑOL»
}

\section{Modesta Salazar Agulló, Emilio A. Martínez Marco y Josep Bernabeu-Mestre}

Universidad de Alicante

\section{RESUMEN}

La investigación aporta un estudio bibliométrico de la Colección de 252 monografías, publicadas entre 1938 y 1964, que aparecieron en la colección Al Servicio de España y del Niño Español. Dicha iniciativa formaba parte del programa de lucha contra la mortalidad infantil y maternal que puso en marcha el régimen franquista, desde sus presupuestos totalitarios y sus políticas pronatalistas.

PALABRAS CLAVE: Estudio bibliométrico; salud materno-infantil; franquismo; España 1938-1964.

MATERNAL AND CHILD HEALTH DURING THE FRANCOISM: BIBLIOMETRIC NOTES ON THE PROGRAM «AL SERVICIO DE ESPAÑA Y DEL NIÑO ESPAÑOL» (IN THE SERVICE OF SPAIN AND THE SPANISH CHILD)

\section{ABSTRACT}

This research comprises a bibliometric study of 252 monographic articles published between 1938 and 1964, which appeared in the collection «In the Service of Spain and the Spanish Child» (Al Servicio de España y del Niño Español). This initiative was part of a programme that sought to combat infant and maternal mortality; it was implemented by Franco's regime and grounded in its totalitarian assumptions and pronatalist policies.

KEY WORDS: Bibliometric study; maternal-infant health; Francoism; Spain 1938-1964.

\section{INTRODUCCIÓN}

Aunque fue en el siglo XVIII cuando empezó a despertarse en la sociedad europea una preocupación por el cuidado del niño, por su educación, por la 
alimentación infantil o la atención a los niños abandonados, sería en las últimas décadas del siglo XIX y primeros decenios del siglo XX cuando se consolidó la autonomía de la pediatría como disciplina separada de la obstetricia y la medicina interna, al mismo tiempo que se iba perfilando la puericultura como especialidad médica encargada de estudiar el conjunto de medios que favorecen el desarrollo físico y psíquico de la infancia ${ }^{1}$. Tanto la puericultura como la especialidad de pediatría fueron una consecuencia lógica del incremento de los saberes en ésta área como de la preocupación social y la sensación de catástrofe que aportaban las elevadas cifras de mortalidad infantil y juvenil. La puericultura desarrollaría cuatro grandes funciones o campos de acción: la higiénica, la médico-sanitaria, la protectora y la educativa. Con la pediatría el organismo del niño sería objeto de atención en sí mismo, sin entenderlo - como sucedía en el modelo tradicional vigente hasta el ochocientos- como un adulto en minoría ${ }^{2}$. En otras palabras se produjo un cambio en la «mirada» al niño, adquiriendo carta de naturaleza propia, ya que dejó de ser considerado como «una etapa de tránsito a la edad adulta» ${ }^{3}$.

El esquema que acabamos de trazar en relación con el desarrollo de la pediatría y la puericultura contemporáneas tuvo su correlato en el caso español, aunque mediatizado por las circunstancias políticas, sociales y económicas que caracterizaron el proceso de modernización en nuestro país ${ }^{4}$ y por las dificultades de institucionalización que mostraron los aspectos sanitarios, y la salud pública en particular ${ }^{5}$. Con todo, a pesar de las dificultades y el retraso con el que se vivieron en la España contemporánea todos estos cambios, la

1 BALlester AÑón, R. (1977), La historia clínica pediátrica durante el siglo XIX: análisis de la ciencia y de la práctica pediátrica a través de las patografias infantiles, Zaragoza, Cátedra de Historia de la Medicina.

2 BAlAguer, E.; BALlester, R. (1995), «La infancia como valor y como problema en las luchas sanitarias de principios de siglo en España», Dynamis, 15, 177-192; BERNABEU MESTRE, J. (1998), «Transición sanitaria y evolución de la medicina (diagnóstico, profilaxis y terapéutica), 1885-1942», Boletín de la Asociación de Demografia Histórica, 16(2), 15-38 (p. 27-28).

3 Perdiguero Gil, E.; Del Cura, M. (2004), «Introducción», en Salvad al niño. Estudios sobre la protección a la infancia en la Europa contemporánea a comienzos del siglo XX, Valencia, Seminari d'Estudis sobre la Ciencia, p. 15-21(p. 21).

4 Martínez Marco, E.; Salazar Agulló, M. (2005), «La salud dels il·licitans fa 100 anys», La Rella, 18, 163-178.

5 Son muchos los trabajos que en los últimos años se han ocupado del desarrollo institucional de la sanidad española contemporánea y de los aspectos relacionados con la salud materno-infantil, entre las últimas novedades podemos destacar por su condición de trabajos de síntesis: Rodríguez Ocaña, E. (2005), Salud Pública en España. Ciencia, profesión y política, siglos XVIII-XX, Granada, Universidad de Granada; y PERDIGUERO, DEL CURA (2004) p. 15-21. 
nueva cultura frente al «niño» tuvo su reflejo en el desarrollo normativo y legislativo, alcanzando la salud materno-infantil un cierto grado de institucionalización ${ }^{6}$.

$\mathrm{Al}$ amparo de las iniciativas legislativas relacionadas con la infancia y de otras como la Instrucción General de Sanidad, publicada en 1904, en el primer tercio del siglo XX se alcanzaron algunos importantes logros en el ámbito de las políticas de salud materno-infantil. Aunque existían antecedentes, como la propuesta de creación en 1910 de un Instituto Nacional de Maternología y Puericultura, o el cierto grado de institucionalización que llegaron a alcanzar las matronas a partir de R.O. de 16 de noviembre de 1888 que disponía las normas que debían regir las carreras de practicante y matrona ${ }^{7}$, fue en 1923 (R.O. de 23 de mayo) cuando se creó en España, un centro docente para la formación de los profesionales encargados de velar por la salud materno infantil: la Escuela Nacional de Puericultura, adscrita al Consejo Superior de Protección a la Infancia, y las Escuelas Provinciales de Puericultura que años más tarde se fueron fundando en diversas capitales de provincia y ciudades importantes, como Valencia, Sevilla o Gijón ${ }^{8}$.

Con todo, habría que esperar a la llegada de la Segunda República y a las reformas sanitarias impulsadas por el gobierno republicano-socialista del primer bienio para que la salud materno-infantil alcanzase un importante grado de desarrollo institucional ${ }^{9}$. El 31 de diciembre de 1931 se publicaba el Decreto de creación de la Sección de Higiene Infantil, dependiente de la Inspección General de Instituciones Sanitarias. El objetivo de la Sección no era

6 En 1904 vería la luz la «Ley de Protección a la Infancia» de Sánchez Guerra, inspirada por el Dr. Tolosa Latour, y cuyo objetivo «era la protección de la salud física y moral de los niños menores de 10 años, control de las casas cuna y lactancia mercenaria, escuelas, etc.». Los contenidos de aquella primera normativa alcanzaron una cierta aplicación a través de la promulgación del Reglamento que la desarrollaba (R.D. de 24 de agosto de 1908), los Reglamentos de Puericultura y Primera Infancia de 1910 y 1916 (RR. DD: de 12 de abril de 1910 y 27 de octubre de 1916), o el Reglamento de Sanidad Provincial de 31 de octubre de 1925. Este movimiento en pro de la infancia partía de dos premisas (PERDIGUERO, DEL CURA (2004), p. 18). En primer lugar que había que sustituir en el imaginario el niño trabajador por el niño escolarizado, y en segundo lugar, había que considerar que la elevadísima morbilidad y mortalidad infantiles era un problema que podía tener solución.

7 Bernabeu Mestre, J.; Gascón PÉrez, E. (1999), Historia de la Enfermería de Salud Pública en España (1860-1977), Alicante, Universidad de Alicante, p. 22-29.

8 BERnABeu, GASCÓN (1999), p. 48-55.

9 Bernabeu-Mestre, J. (2000) «La utopía reformadora de la Segunda República: la labor de Marcelino Pascua al frente de la Dirección General de Sanidad, 1931-1933», Rev Esp Salud Pública, 74, 1-13. 
otro que el de luchar contra la mortalidad infantil y aspectos con ella relacionados. Contaba con los departamentos de mortalidad materna, de mortinatalidad, de higiene prenatal y preescolar. Además, a través de una orden ministerial de 30 de marzo de 1932, se creaban en todos los institutos provinciales de higiene, servicios de higiene infantil que debían contar con consultas de higiene prenatal, de lactantes y de higiene escolar, una propuesta asistencial que se trasladaría a los centros secundarios de higiene y a los centros de higiene rural ${ }^{10}$. Todas estas iniciativas respondían al desarrollo de unas políticas de salud encaminadas a lograr la colectivización de la asistencia médica, primando la voluntad preventivista que debía orientar toda la acción sanitaria y buscando la necesaria coordinación entre sanidad (salud pública), prevención social y asistencia sanitaria pública ${ }^{11}$.

El golpe de estado de 18 de julio de 1936 y la contienda civil que desencadenó, truncaron el desarrollo de la propuesta de modelo de asistencia médica que se había trazado durante la Segunda República, aunque se mantuviese una cierta inercia institucional al respetar buena parte de las estructuras y servicios que conformaban la sanidad nacional ${ }^{12}$. En el ámbito de la salud materno-infantil, y a través del programa Al Servicio de España y del Niño Español ${ }^{13}$, el nuevo régimen, desde los parámetros totalitarios que lo caracte-

10 Bernabeu; Gascón (1999), p. 38-39.

11 Bernabeu Mestre, J. (2003), «La contribución del exilio científico español al desarrollo de la salud pública venezolana: Santiago Ruesta Marco (1938-1960)», en BARONA, J. LL. (ed) Ciencia, salud pública y exilio (España 1875-1938, Valencia, Seminari d'Estudis sobre la Ciencia, p. 223-256 (p. 230).

12 Las reformas iniciadas por los gobiernos del primer cuarto de siglo fueron encaminadas a conseguir la creación de un Ministerio de Sanidad y un Seguro Obligatorio de Enfermedad, liderado por Marcelino Pascua, entre otros, pero la derrota republicana en la Guerra Civil impidió la puesta en práctica de esas políticas sanitarias (MARSET CAMPOS, P.; SÁEZ GómEZ, J. M.; Martínez NaVArro, F (1995), «La Salud Pública durante el franquismo», Dynamis, 15, 211-250 (p. 217-218)). Tal y como figura en el preámbulo de la Ley General de Sanidad aprobada en 1986 «es, en efecto, un dato histórico fácilmente verificable que las respuestas públicas al reto que en cada momento ha supuesto la atención a los problemas de salud de la colectividad han ido siempre a la zaga de la evolución de las necesidades sin conseguir nunca alcanzarlas, de manera que se ha convertido en una constante entre nosotros la inadaptación de las estructuras sanitarias a las necesidades de cada época».

13 Este programa ha sido objeto de atención monográfica en investigaciones llevadas a cabo por Josep Bernabeu y Enrique Perdiguero (Bernabeu Mestre, J.; Perdiguero Gil, E. (2001) «At the Service of Spain and Spanish Children: Mother and Child Healthcare in Spain During the First Two Decades of Franco's Regime (1939-1963)», in LöWY, I.; KRIGE, J. (eds) Image of Disease. Science, Public Policy and Health in Post-war Europe, Luxembourg, Offi- 
rizaban ${ }^{14}$ y en el marco de una ideología netamente poblacionista (su objetivo era que España alcanzara los 40 millones) ${ }^{15}$, situó como objetivo prioritario la reducción de la mortalidad infantil y juvenil, la mortinatalidad y la mortalidad materna ${ }^{16}$. Al análisis de las publicaciones que generó dicho programa hemos dedicado el trabajo de investigación que presentamos a continuación.

ce for Official Publications of the European Communities, p. 167-186; BERnABEU-Mestre, J. (2002), «Madres y enfermeras. Demografía y salud en la política poblacionista del primer franquismo, 1939-1950», Revista de Demografia Histórica, 20 (1), 123-143), nuestro trabajo se inserta dentro de dicha línea de investigación y pretende llevar a cabo un análisis sistemático de las publicaciones que se generaron. El análisis bibliométrico supone un primer paso.

14 En relación con la problemática infantil, el papel de las mujeres y la ideología totalitaria del régimen franquista, en los últimos años se ha producido un creciente interés historiográfico. Entre otras publicaciones podemos citar las de PALACIO LIS, I. (2003), Mujeres ignorantes: madres culpables. Adoctrinamiento y divulgación materno infantil en la primera mitad del siglo XX, Valencia, Universitat de València; VINYES, R.; ARMENGOU, M.; BELIS, R. (2005), Los niños perdidos del franquismo, Barcelona, RBA; AMICH EliAs, C. (2005), El poder y los derechos del niño en el franquismo, Salamanca, Ediciones de la Universidad de Salamanca; o Cenarro, A. (2006) La sonrisa de Falange: Auxilio Social en la guerra civil y la posguerra, Barcelona, Critica.

15 NASH, M. (1996) «Pronatalismo y maternidad en la España franquista», en Bock, G.; Thane, P. (eds) Maternidad y políticas de género: la mujer en los estados de bienestar europeos, 1880-1950, Madrid, Cátedra, p. 279-308.

16 En el contexto de la transición demográfica española, las décadas centrales del siglo $\mathrm{XX}$, han sido consideradas un momento clave en el descenso de la mortalidad infantil (GómEZ REDONDO, R. (1992), La mortalidad infantil española en el siglo XX. Madrid, Centro de Investigaciones Sociológicas, p. 101). Tras iniciar el siglo con tasas de mortalidad infantil por encima del 200 por mil en el conjunto nacional, en la década de 1930 la mortalidad infantil española se había reducido a un 123 por mil. Superado el paréntesis de los años inmediatos a la posguerra, la situación mejoró, pero en materia de mortalidad infantil, y más particularmente en mortalidad postneonatal, España ostentaba, para el contexto de la Europa occidental y el período 1941-1950, las segundas tasas más elevadas tras Portugal (Arbelo Curbelo, A. (1954), La mortalidad postneonatal en España (Fallecidos de 1 a 11 meses), 1941-1950, Madrid, Dirección General de Sanidad («Al Servicio de España y del niño español», núm. 195), p. 18). Con todo, aunque las décadas centrales del siglo XX se caracterizaron por ser en su conjunto uno de los períodos donde más se avanzó en la reducción de la mortalidad infantil, las diferencias regionales, como consecuencia de las desigualdades en salud, lejos de corregirse se acentuaron (BERNABEUMestre, J.; Caballero Pérez, P.; Galiana Sanchez, M.E; Nolasco Bonmatí, A (2005), «Niveles de vida y salud en la España del primer franquismo: las desigualdades en la mortalidad infantil», Revista de Demografia Histórica, 24 (1/2), 173-193. 


\section{MATERIAL Y MÉTODO}

Como acabamos de indicar, el objetivo del trabajo es realizar una aproximación bibliométrica a la colección de publicaciones Al servicio de España y del niño español. Se trata de una investigación de carácter heurístico previa y necesaria para poder profundizar, en una segunda etapa, en el análisis de los fines, actividades e iniciativas de todo tipo que se generaron en el marco de aquel programa de salud materno-infantil.

Para la localización de las publicaciones que fueron apareciendo se han consultado los catálogos y las bases de datos siguientes: Biblioteca Nacional de Madrid, Biblioteca-Archivo de la Real Academia Nacional de Medicina de Madrid, Biblioteca Nacional de Ciencias de la Salud (Escuela Nacional de Sanidad de Madrid) y el Catálogo Colectivo de las Bibliotecas Universitarias Españolas.

Con la información recogida se creó una base de datos con el programa EXCEL para Windows 2000, en el que se recogieron las siguientes variables: autor/es, título, número de la publicación, mes y año, extensión de la obra, tamaño y biblioteca, catálogo, o repertorio donde se ha localizado, además del consiguiente apartado de observaciones.

Las palabras clave, seleccionadas a partir del título de las publicaciones, han permitido elaborar un listado de las principales áreas temáticas que fueron objeto de atención o merecieron el interés del programa. Junto al análisis de las principales temáticas, se procedió a cuantificar el número de obras por autor, el índice de firmantes por obra, o la evolución temporal de las publicaciones aparecidas, entre otras cuestiones.

El trabajo se ha completado con un anexo donde aparecen por orden correlativo las diferentes publicaciones que fueron apareciendo, con indicación de la biblioteca, catálogo, repertorio o publicación donde se han localizado.

\section{RESULTADOS}

\section{DESCRIPCIÓN DE LA COLECCIÓN}

Se han localizado un total de 311 números publicados, correspondiendo el $\mathrm{n}^{\mathrm{o}} 1$ al mes de enero del año 1938 y el último al de enero de 1964. Cada año y de manera ininterrumpida se publicaron 12 números, salvo el año 1940 que solo aparecieron 10. No se encontró ninguna referencia que informe de los contenidos y características de los números 307 y 310 . 
No tenemos constancia de la tirada de cada publicación. Al parecer inicialmente iba destinado a los profesionales de los centros sanitarios creados con el programa, aunque a partir de 1951 sí consta que hay suscripciones previo pago (probablemente contra reembolso). Tampoco tenemos noticia del precio inicial de los ejemplares, ya que no aparece ninguna mención a este aspecto hasta el número 155 (Enero 1951) ${ }^{17}$.

La colección se caracteriza por una constancia en el formato. Los documentos están escritos a doble página, a un espacio y por término medio tiene unas 330 palabras mecanografiadas por página. Sus medidas son de tamaño cuartilla ( $21 \times 15 \mathrm{~cm}$.), con una portada que no se modificó apenas en el cuarto de siglo que mantuvo de vigencia. En el centro de la misma siempre aparece en mayúsculas el título de la monografía, el autor de la obra (y en ocasiones el cargo). En la parte superior suele figurar el texto Publicaciones al Servicio de España y del Niño Español, junto con el año (con números romanos), el mes y el número de la colección. En la parte inferior suele aparecer el organismo editor.

Los textos fueron editados por el Ministerio de Gobernación, de quien dependía la Dirección General de Sanidad. En algunas de las publicaciones aparecen como responsables de la edición organismos como los Servicios Centrales de Higiene Infantil, o la Sección de Maternología, Puericultura e Higiene. En cualquier caso, se trataba de servicios que mantenían una dependencia jerárquica de la Dirección General de Sanidad.

Como tendremos ocasión de comentar, en muchas ocasiones las monografías respondían a autorías colectivas bajo la denominación de un Comité de Redacción. Son diversas las publicaciones que recogen la estructura organizativa del programa, así como las funciones de los servicios y de los profesionales que los conformaban (maternólogos, puericultores, matronas, enfermeras, etc.), o los logros obtenidos con el mismo. Estas últimas informaciones hay que contextualizarlas en el carácter de propaganda política del régimen franquista que cabe a atribuir a la Colección Al servicio de España y del niño español.

Como era previsible, no hay colores en ninguna publicación, pero sí mapas, tablas, planos, además de la reproducción de algunos de los carteles de

17 En el número 155 aparece una nota en color verde con el siguiente texto: «Aviso a los suscriptores: A partir de 1 de Enero de 1951, el precio de la suscripción anual a las publicaciones 'Al servicio de España y del niño español' es de 50 Pesetas. El importe de la suscripción correspondiente a dicho año, se cobrará contra reembolso al recibir el número correspondiente al mes de febrero próximo». 
divulgación sanitaria con mensajes educativos dirigidos fundamentalmente a las madres, que se publicaron en aquellos años ${ }^{18}$.

El primer número de la Colección (enero de 1938) lleva por título La protección de los niños huérfanos abandonados e indigentes de Sevilla y el número 2 continúa la temática con una monografía sobre Lo que ha hecho Sevilla por los niños desde que se inicio el glorioso Movimiento Nacional, ambos trabajos están firmados por el Dr. José Luis Morales. El último número localizado con autoría (311 de enero de 1964) está firmado por el Dr. Adolfo Serigó Segarra y lleva por título La evolución de la mortalidad infantil en España.

La Colección está compuesta por 311 números que se corresponden con 252 monografías. De ellas, doscientas, es decir el $64,39 \%$ tienen un carácter mensual y cuarenta y siete de ellas (el 30,2\%) reúnen la condición de número doble al aparecer con carácter bimensual. Las cinco monografías restantes $(5,4 \%)$ se presentaron bajo la fórmula de número trimestral o cuatrimestral. El primer número triple (84-85-86) corresponde a los primeros meses de 1945, fue preparado por el Comité de Redacción y lleva por título Servicios de Sanidad Infantil y Maternal (Memoria del año 1944). Hay dos publicaciones más de estas características, las correspondientes a los números 100-101-102 y 123-124-125. Las publicaciones cuatrimestrales corresponden a los números 109 a 112 y 257 a 260. La primera apareció en marzo de 1947. Llevaba por título El niño español en el siglo $X X$ y su autor fue el doctor Juan Bosch Marín. La segunda, obra del Comité de Redacción, apareció en 1959, con el título de Congreso Internacional de Matronas.

Al no haber podido disponer de los volúmenes originales y haber tenido que recurrir a fuentes secundarias con diferentes sistemas de catalogación, no tenemos la certeza de que algunos datos como el número de páginas o el mes de publicación sea coincidente en todas ellas. Por tanto, con las precauciones que acabamos de señalar, los ejemplares más voluminosos corresponden al 51-52 y al 153-154. En el primer caso se trata de una monografía publicada por Villar Salinas sobre la Natalidad contemporánea en España, trabajo por el que recibió el premio Conde de Toreno. El segundo caso, hace referencia al trabajo publicado por Enrique Bravo Sánchez del Peral, donde se compendia la organización de los servicios de salud materno-infantil, la legislación vigente, y diversas cuestiones como el reglamento de los Centros Maternales de Urgencia, los «Modelos de impresos oficiales» o el «Diploma a la madre

18 Entre los catálogos y bases de datos consultadas, las descripciones más detalladas son las que ofrece la Biblioteca Nacional, al hacer constar también el número de hojas anexas, plegadas, mapas, láminas, etc. 
ejemplar». Dicho volumen, nos permitió, además, localizar un grupo importante de publicaciones que no aparecían en los catálogos y bases de datos consultados.

En relación con el lugar de publicación, hay que indicar que todos los números fueron publicados en Madrid, salvo los primeros números (hasta el 15) en los que figura la leyenda Impreso en Valladolid, y que corresponden, lógicamente, a los años de la guerra civil.

\section{LAS TEMÁTICAS DE LAS PUBLICACIONES}

A partir de los títulos que aparecen en las 252 monografías, hemos seleccionado una relación de palabras claves que ha permitido su clasificación por grupos temáticos. A efectos estadísticos cada publicación ha sido incorporada únicamente a un grupo temático, priorizando la palabra clave que se ha considerado principal. En la siguiente tabla figuran los grupos realizados y la distribución de frecuencias, así como el número de colección de las publicaciones que se han incluido en cada grupo.

El grupo temático más voluminoso es el relativo a «Administración Sanitaria». En el mismo se han incluido las publicaciones relacionadas con la organización, planificación, legislación y administración sanitarias, las actividades hospitalarias, las memorias de actividades, así como temas de política sanitaria o social, seguros sociales y mutualidades.

El segundo grupo de temas en importancia corresponde a «Asistencia Sanitaria» e incorpora toda la temática asistencial, incluyendo enfermedades infecciosas y vacunaciones (poliomielitis, lepra, tuberculosis, paludismo, etc.) y otras como raquitismo, reumatismo y corea. Nueve publicaciones tratan de la discapacidad física, invalidez y rehabilitación, lógico tras una contienda civil y una época de penuria en todos los campos. Se incluyen ocho publicaciones relacionadas con psiquiatría, psicología y salud mental. También se han incluido publicaciones relacionadas con el parto y la asistencia al lactante.

El tercer apartado en frecuencia, exceptuando el de varios, es el denominado «Análisis demográfico». Se abordan los indicadores demográficos y sanitarios correspondientes a las diferentes etapas evolutivas del niño (primer año, post-neonatal, lactante prematuro, preescolar), tanto a nivel nacional, como en estudios regionales o locales (Salamanca, Sevilla, Las Palmas, Zaragoza, Madrid, medio rural). La problemática maternal también es abordada. 
MODESTA SALAZAR AGULLÓ, EMILIO A. MARTÍNEZ MARCO Y JOSEP BERNABEU-MESTRE

TABLA 1: GRUPOS TEMATICOS

\begin{tabular}{|c|c|c|c|}
\hline GRUPO & $\begin{array}{l}\text { NÚMERO DE } \\
\text { MONOGRAFÍAS }\end{array}$ & $\%$ & $\begin{array}{l}\text { NÚMERO DE LAS PUBLICACIONES INCLUIDAS EN } \\
\text { CADA EPÍGRAFE }{ }^{\text {a }}\end{array}$ \\
\hline $\begin{array}{l}\text { ADMINISTRACION } \\
\text { SANITARIA }\end{array}$ & 62 & 24,6 & $\begin{array}{l}3,16,18,19,25,28,31,33,39,40,41,43,45, \\
57,66,69,70,71,72,95,98,99,104,(117--118), \\
122,127,130,131,138,148,159,160,161,162, \\
170,(181-182), 185,192,193,194,, 196,(197- \\
198), 207,208,210,218,(225-226), 227,228, \\
229,231,232,235,236,(237-238), 239,250, \\
(266-267),(281-282), 283,290,(302-303)\end{array}$ \\
\hline $\begin{array}{l}\text { ASISTENCIA } \\
\text { SANITARIA }\end{array}$ & 49 & 19,5 & $\begin{array}{l}7,27,37,42,48,54,58,(63-64), 75,(84-85-86), \\
87,88,(93-94), 97,(100-101-102), 126,(128- \\
129),(136-137), 142,145,149,150,(153-154), \\
156,158,166,171,(173-174), 178,183,(186- \\
187), 190,191,211,215,217,230,(242-243), \\
247,(253-254), 262,(275-276),(278-279),(287- \\
288), 289,293,(294-295), 301,(305-306)\end{array}$ \\
\hline $\begin{array}{l}\text { ANÁLISIS } \\
\text { DEMOGRÁFICO }\end{array}$ & 30 & 11,9 & $\begin{array}{l}5,15,17,20,29,50,(51-52), 55,62,68,77,(80- \\
81), 96,(115-116),(133-134), 139,144,146,155, \\
157,172,195,201,219,224,251,255,311\end{array}$ \\
\hline $\begin{array}{l}\text { FORMACIÓN Y } \\
\text { PROPAGANDA }\end{array}$ & 27 & 10,7 & $\begin{array}{l}8,11,14,(23-24), 32,35,44,53,59,73,89,90, \\
91,103,121,135,(140-141), 152,163,184,214, \\
240,241,261,284,(291-292), 298\end{array}$ \\
\hline $\begin{array}{l}\text { SALUD } \\
\text { INTERNACIONAL }\end{array}$ & 19 & 7,5 & $\begin{array}{l}47,(123-124-125), 175,176,199,202,203,(204- \\
205),(221-222),(233-234),(245-246),(248-249), \\
252,256,(257-258-259-260),(263-264), 271, \\
(285-286),(308-309)\end{array}$ \\
\hline HIGIENE & 16 & 6,3 & $\begin{array}{l}4,6,9,13,56,60,61,76,83,92,(107-108) \\
(113-114), 180,206,220,244\end{array}$ \\
\hline $\begin{array}{l}\text { NUTRICION, } \\
\text { ALIMENTACIÓN Y } \\
\text { DIETÉTICA }\end{array}$ & 12 & 4,8 & $\begin{array}{l}10,12,21,36,38,46,49,(119-120), 151,164 \\
165,213\end{array}$ \\
\hline VARIOS & 37 & 14,7 & $\begin{array}{l}1,2,22,26,30,34,65,67,78,79,82,(105-106), \\
(109-110-111-112), 132,143,147,(167-168), \\
169,177,179,(188-189), 200,209,216,223, \\
265,268,(269-270),(272-273), 274,277,280, \\
(296-297),(299-300), 304,307,310\end{array}$ \\
\hline
\end{tabular}

${ }^{a}$ Para la localización de las publicaciones véase el anexo. Entre paréntesis figuran las monografías de más de un número.

El siguiente apartado en frecuencia se corresponde con el de «Formación y propaganda», e incorpora aquellas publicaciones cuyo título sugería tanto la 
formación del personal especializado como el enfoque educativo dirigido a la población en general (propaganda). Se presentan programas de sanidad o conferencias, cursos y cursillos de puericultura, además de hacer referencia a los medios audiovisuales (radio y cine) como medios de divulgación de conocimientos higiénicos y sanitarios.

El apartado denominado «Salud Internacional» incluye publicaciones relacionadas con el papel de la UNICEF y la OIT, y aportaciones de autores españoles a foros internacionales. Este último aspecto, reúne una cierta relevancia si atendemos al aislamiento internacional que sufrió el régimen franquista en los años objeto de estudio.

La «Higiene» ocupa también un lugar destacado en las publicaciones (el $6,3 \%$ de las mismas). Los textos abordan problemáticas relacionadas con la higiene escolar, bucal, mental, del embarazo, así como descripciones de la labor de los dispensarios de higiene.

El apartado de «Nutrición, alimentación y dietética», con un $4,8 \%$ de las publicaciones, contiene trabajos dirigidos fundamentalmente al período de la lactancia.

Por último, en el apartado de «Varios» se han incluido publicaciones muy diversas. Ocho de ellas versan sobre aspectos relacionados con la «moralidad» y otras ocho están relacionadas con el arte y la infancia.

Por otra parte, el análisis semántico de los títulos de las publicaciones pone de manifiesto que la familia de palabras con raíz infancia, es la que más se repite (78 títulos, el 30,8\%), seguido de la familia maternal/madre con $39(15,4 \%)$. Niño/a/s se repite en 33 con el 13\% y mujer aparece sólo en 3 casos (1,2\%).

\section{PRODUCTIVIDAD DE LOS AUTORES QUE FIRMARON LAS PUBLICACIONES}

El número final de monografías localizadas (independientemente de los números de la Colección que encerraba cada una de ellas) fue de 252 . Treinta y siete de las mismas (el 14,7\%) aparecen firmadas por el Comité de redacción; del resto de publicaciones hay que destacar que más de tres cuartas partes son obra de un único firmante.

Los treinta y siete trabajos atribuidos al Comité de Redacción se publicaron entre principios de 1941 (num. 35) y mediados de 1963. Su producción consta esencialmente de programas, memorias, legislación, un texto denominado Charlas radiofónicas de puericultura, textos sobre acuerdos internacionales o efemérides como Las bodas de plata del Instituto de Puericultura de Gijón.

Si descontamos los 37 trabajos firmados por el Comité de Redacción, siete autores firman 86 de las publicaciones restantes, es decir el 34,1\%, o dicho de 
otro modo, casi la mitad de las obras están firmadas por estos autores y el Comité de Redacción (ver la tabla 2).

TABLA 2: PRODUCTIVIDAD DE LOS PRINCIPALES AUTORES ${ }^{19}$

\begin{tabular}{|l|c|c|c|}
\hline AUtOR & $\begin{array}{c}\text { NÚMERO } \\
\text { PUBLICACIONES }\end{array}$ & $\begin{array}{c}\text { PORCENTAJE SOBRE } \\
\text { EL TOTAL }\end{array}$ & $\begin{array}{c}\text { PORCENTAJE ACU- } \\
\text { MULADO }\end{array}$ \\
\hline Comité de Redacción & 37 & 14,7 & 14,7 \\
\hline Juan Bosch Marín & 32 & 12,7 & 27,4 \\
\hline Antonio Arbelo Curbelo & 14 & 5,6 & 33,0 \\
\hline Manuel Blanco Otero & 14 & 5,6 & 38,6 \\
\hline Bravo Sánchez del Peral & 9 & 3,6 & 42,2 \\
\hline José Selfa & 7 & 2,8 & 45,0 \\
\hline Ruiz Santamaría & 5 & 2,0 & 47,0 \\
\hline Villar Salinas & 5 & 2,0 & 49,0 \\
\hline
\end{tabular}

Los autores que más publicaron en esta colección fueron Juan Bosch Marín, Antonio Arbelo Curbelo y Manuel Blanco Otero quienes, en conjunto, suscribieron más del 23,8\% de la producción (60 monografías).

El Dr. Bosch Marín (Carlet (Valencia) 12-9-1902-Madrid, 17-1-1995) fue el autor más prolífico ya que figuró como firmante único en 23 monografías $(9,1 \%)^{20}$. Además firmó otras muchas como coautor y prologó bastantes de las monografías. La temática que abordó fue muy variada: política familiar y sanitaria, cuestiones de política demográfica con un trabajo titulado Cómo ha

19 Esta producción está compuesta de los artículos firmados individualmente más las aportaciones realizadas con otros autores. Por ejemplo el Dr. Bosch firmó en solitario 23 monografías y 9 como coautor.

20 Concretamente el primer y último número de la Colección en el que aparece como único firmante son el número 3, Problemas de maternología y puericultura, (aparecido en marzo de 1938), y el número 299-300, sobre Necesidades biológicas de la infancia, aparecido en febrero de 1962. Pediatra de origen valenciano, terminada la guerra obtuvo por Concurso la plaza de Jefe de los Servicios de Higiene Infantil de la Dirección General de Sanidad. Se le consideró inspirador de la Ley de Sanidad Maternal e Infantil de 1941. También ocupó la plaza de Jefe de la Obra Maternal e Infantil del Instituto Nacional de Previsión, además de miembro de la Real Academia de Medicina (Bosch MARIN, J. (1947), El niño español en el siglo XX: Discurso para la recepción pública del Académico Electo Excmo. Sr. Dr. D. Juan Bosch Marín y contestación del Académico Numerario Excmo Sr. Dr. D. José A. Palanca, Madrid, Gráficas González.). Fue presidente durante 8 años de la Asociación Española de Médicos, escritores y artistas (1945-1952) (http:www.medicosescritoresyartistas.com/). 
resuelto la Italia de Mussolini el problema demográfico, maternología y puericultura, y problemas relacionados con enfermedades infecciosos como la difteria o la poliomielitis.

La firma de Manuel Blanco Otero se constata en catorce monografías (en la mitad de las cuales figura como autor único). Sus trabajos abarcan desde el número 55 de la publicación hasta el 293 (Septiembre de 1942 a Julio de 1962). Publicó dos trabajos sobre UNICEF y otros dos relacionados con la radio como medio de divulgación de conocimientos higiénicos y sanitarios ${ }^{21}$. El resto de su producción es variada aunque destacamos el número 244 sobre Higiene mental infantil y centros de Child Guidance. En 1954 figura como Redactor-Jefe de la Colección.

El Dr. Antonio Arbelo Curbelo (Las Palmas, 1909- Madrid, 2004), firmó trece trabajos en solitario y uno junto a Dionisio Morcillo (Num.113-114, Bases para una crítica de la labor dispensarial de higiene infantil). Su producción se situó entre los números 80 y 255 de la Colección, lo que corresponde al periodo 1944-1959. Sus monografías reflejaban una preocupación por los temas relacionados con la mortalidad infantil y juvenil ${ }^{22}$.

Hubo otros autores destacados, como Enrique Bravo Sánchez del Peral, quien figura en gran parte de las obras como «Administrador». Este autor publicó en nueve ocasiones, dos de ellas como coautor. La temática de sus monografías guardaba relación con los temas de índole organizativa y legislativa de la sanidad infantil y maternal. El primer trabajo que publicó correspondía al número 153 de la colección y el último al número 239 (1950 y 1957, respectivamente).

Por su parte, el Dr. D. José Selfa publicó en solitario siete monografías, entre 1941 y 1958. Sus trabajos versaron sobre dietética, paludismo, reumatismo y otros problemáticas referidas a la provincia de Valencia. En 1948 publicó la monografía titulada Asistencia hospitalaria infantil en los EEUU: impresiones de un viaje de estudios (124-125-126).

21 En concreto en el número 223, Tres diálogos sobre la salud (entrevista radiofónica con el doctor J. Mateo) y el 241 sobre La medicina en la radio.

22 Canario de origen, estudió en Santiago de Compostela la carrera de medicina y ejerció la actividad profesional en su ciudad natal, Las Palmas de Gran Canaria, tras el regreso del servicio militar en Guinea Ecuatorial. Posteriormente se instaló en Madrid. Se doctoró en la Universidad Complutense de Madrid a los 34 años y concursó en diversas oposiciones. Médico puericultor del Estado, fue Asesor de Demografía en la Dirección General de Sanidad, y profesor de Estadística y Demografía en la Escuela Nacional de Puericultura. Estuvo vinculado a la Asociación Española de Pediatría, como secretario y vicepresidente (HERRERA HERNÁNDEZ, M. (2004), «In memoriam Dr. Antonio Arbelo Curbelo», Boletín de la Sociedad Canaria de Pediatría, 28 (1), 7-10). 
Por último, en la relación de autores que más trabajos publicaron, hay que mencionar a los doctores José Antonio Ruiz Santamaría y Jesús Villar Salinas, con cinco monografías cada uno.

Con relación a la participación femenina, en la tabla 3 queda reflejada dicha contribución. Las obras firmadas por mujeres representan el 5,2\%. Como puede apreciarse, en sus trabajos se abordan cuestiones relacionadas con actividades femeninas, la puericultura, el servicio social o las matronas.

TABLA 3: AUTORAS QUE PUBLICARON EN LA COLECCIÓN.

\begin{tabular}{|c|c|c|}
\hline NOMBRE Y APELLIDOS & ACTIVIDAD PROFESIONAL & TITULO \\
\hline $\mathrm{D}^{\mathrm{a}}$ Carmen Isern y Galceran & $\begin{array}{l}\text { Inspectora enseñanza } \\
\text { primaria }\end{array}$ & $\begin{array}{l}\mathrm{N}^{\mathrm{o}} 128-129 \text { La mujer en la vida } \\
\text { del trabajo. Su misión social, su } \\
\text { aspecto jurídico } \\
\mathrm{N}^{\mathrm{o}} 231 \text { La salud y la psicología } \\
\text { del escolar }\end{array}$ \\
\hline Victoria Losada & Pediatra & $\begin{array}{l}\text { o 272-273 La mujer y la pedia- } \\
\text { tría }\end{array}$ \\
\hline $\begin{array}{l}\text { María García Martín y } \\
\text { Julieta Samaniego Berlanga }\end{array}$ & Matronas & $\begin{array}{l}\mathrm{N}^{\circ} 202 \text { La participación españo- } \\
\text { la en el Congreso Internacional } \\
\text { de Matronas, de Londres }\end{array}$ \\
\hline $\begin{array}{l}\text { María Raquel Payá Ibars } \\
\text { Matilde Fernández de } \\
\text { Henestrosa; Sor Montserrat } \\
\text { Pedret; Da Carmen Werner. }\end{array}$ & Servicio social & $\begin{array}{l}\mathrm{N}^{\mathrm{o}} 278-279 \text { El servicio social y la } \\
\text { inadaptación de la infancia y de } \\
\text { la juventud }\end{array}$ \\
\hline $\begin{array}{l}\text { Srta. Rosario Rodríguez } \\
\text { Babe }\end{array}$ & Servicio social & $\begin{array}{l}\mathrm{N}^{\circ} 26 \text { Nuevas orientaciones al } \\
\text { trabajo social femenino }\end{array}$ \\
\hline $\begin{array}{l}\text { Matilde Fernández de } \\
\text { Henestrosa }\end{array}$ & Servicio social & $\begin{array}{l}\mathrm{N}^{\circ} 233-234 \text { Información sobre el } \\
\text { «social case work» (resumen del } \\
\text { seminario... Madrid, 1956) }\end{array}$ \\
\hline $\begin{array}{l}\text { Rosario Rodríguez Encinas, } \\
\text { Antonia Hernández }\end{array}$ & Matronas & $\begin{array}{l}\mathrm{N}^{\circ} 209 \text { Moral profesional y labor } \\
\text { social de la matrona }\end{array}$ \\
\hline $\begin{array}{l}\text { Matutina Rodríguez Álvarez } \\
\text { de Torner }\end{array}$ & $\begin{array}{c}\text { Jefa del Servicio Provincial } \\
\text { de Puericultura Asturias }\end{array}$ & $\begin{array}{l}\mathrm{N}^{\circ} 22 \text { La puericultura es a la vez } \\
\text { ciencia y arte. }\end{array}$ \\
\hline $\begin{array}{l}\text { Doña Irene Rogi Acuña, } \\
\text { viuda de Martínez Anido }\end{array}$ & No consta & $\begin{array}{l}\mathrm{N}^{\circ} 43 \text { Preservación de la infan- } \\
\text { cia contra la tuberculosis (obra } \\
\text { Brancher) }\end{array}$ \\
\hline $\begin{array}{l}\text { Juliana Bueno y Julia Soler } \\
\text { Sáez }\end{array}$ & $\begin{array}{c}\text { Matronas } \\
\text { Presidentas de los Colegios } \\
\text { de Barcelona y Valencia } \\
\end{array}$ & $\begin{array}{l}\mathrm{N}^{\circ} 211 \text { La matrona ante los } \\
\text { seguros sociales. }\end{array}$ \\
\hline Basilia Cuerdo & No consta & $\begin{array}{l}\text { No 281-282 La asistencia social y } \\
\text { el servicio psiquiátrico infantil }\end{array}$ \\
\hline $\begin{array}{l}\text { Carmen Díaz y Mariana } \\
\text { Gómez }\end{array}$ & Matronas & $\begin{array}{l}\text { № } 210 \text { La matrona en el servicio } \\
\text { rural }\end{array}$ \\
\hline
\end{tabular}




\section{DISCUSIÓN Y CONCLUSIONES}

Los resultados de la investigación han puesto de manifiesto la continuidad que tuvo, a través de su Colección de publicaciones, el programa de salud materno-infantil Al Servicio de España y del Niño Español. Entre 1938 y 1964 se han podido localizar 311 números que se corresponden con 252 monografías.

El análisis bibliométrico nos ha permitido conocer las temáticas que se abordaron y su frecuencia, además de detectar el núcleo y las características de los principales productores.

En el capítulo de las problemáticas abordadas, destaca el predominio que muestran las cuestiones asistenciales frente a las de carácter preventivo. Los temas relativos a la higiene son más frecuentes en los primeros números $(4,6$, $13,56)$ y a lo largo de la evolución de la Colección van siendo reemplazados por otras problemáticas de carácter más asistencial. Aunque parece obligado esperar a la lectura y análisis de los textos para poder emitir un juicio más definitivo, no parece exagerado apuntar que dicho predominio obedece al cambio de política sanitaria que se acabó imponiendo en el franquismo y que condujo a primar las tareas asistenciales de atención a la enfermedad ${ }^{23}$. Tampoco parece descabellado subrayar, a la luz de los resultados, la existencia de una cierta dicotomía entre el enfoque pediátrico o el puericultor que se le podía dar al programa.

En el apartado de los productores, junto con el anónimo Comité de Redacción, responsable del $14,7 \%$ de las publicaciones, siete autores firman el $34,3 \%$ de las monografías. Es decir, casi la mitad, un $49 \%$ de las publicaciones, responden a la autoría de un reducido grupo de autores, ya que muy probablemente los máximos productores (los doctores Bosch Marín, Blanco Otero, Arbelo Curbelo, Bravo Sánchez del Peral, Selfa, Ruiz Santamaría y Villar Salinas) formaron parte del Comité. Conviene destacar esta circunstancia, por la influencia que pueda tener en el momento de perfilar la uniformidad que pudo guiar los presupuestos conceptuales, metodológicos e ideológicos que guiaban el programa Al Servicio de España y del Niño Español ${ }^{24}$.

23 Bernabeu, Gascón (1999), p. 95.

24 Como ya se ha indicado, algunos de estos aspectos han sido abordados parcialmente en investigaciones previas llevadas a cabo desde los Departamentos de Enfermería Comunitaria, Medicina Preventiva y Salud Pública e Historia de la Ciencia y de Salud Pública de las Universidades de Alicante y Miguel Hernández de Elche (BernabeU, Perdiguero (2001); BerNABEU (2002); BERNABEU et al (2006). 
Como indicábamos al plantear el objetivo de la investigación, nuestro trabajo, de carácter fundamentalmente heurístico y descriptivo, tiene la condición de paso previo para poder abordar, a partir del estudio sistemático y pormenorizado de las publicaciones localizadas, un análisis en profundidad del contenido del programa de salud materno infantil implementado durante el primer franquismo. El interés que puede mostrar dicha tarea investigadora radica en la importancia que tuvo el período objeto de análisis desde el punto de vista de la consolidación de la transición sanitaria y epidemiológica de la población española ${ }^{25}$, en el importante componente ideológico que acompañó el discurso científico que sustentaba el programa ${ }^{26}$ y que se vio igualmente reflejado en otras actuaciones sanitarias y parasanitarias del régimen franquis$\mathrm{ta}^{27}$, en el no menos importante momento histórico que representaron las décadas de los años de 1940, 1950 y 1960 con la influencia que tuvieron los cambios de orientación de las políticas de salud ${ }^{28}$, o en el impacto de la terapéutica antibiótica y los programas de vacunación en las estrategias encaminadas a abordar los problemas de salud materno infantil ${ }^{29}$.

\footnotetext{
25 BERNABEU et al (2006).

26 BERNABEU (2002).

27 Pueden consultarse, entre otros trabajos: RuIz SOMAVILLA, M.J.; JIMÉNEZ LuCENA, I. (2001) «Un espacio para mujeres. El Servicio de Divulgación y Asistencia Sanitario-Social en el primer franquismo», Historia Social, 39, 67-85; JimÉnez Lucena, I.; Ruiz Somavilla, M.J.; CASTEllanos GuerRero, J: (2002), «Un discurso sanitario para un proyecto político. La educación sanitaria en los medios de comunicación de masas durante el primer franquismo», Asclepio, 54(1), 201-218; ECHEVERri DÁVILA, B (2003), «La protección de la infancia: la educación sanitaria de las madres en la posguerra española», Historia y política, 9, 279-308.

28 MARSET, SÁEZ, MARTíNEZ (1995).

29 BERNABEU et al (2006).
} 


\title{
ANEXO: RELACIÓN DE LAS PUBLICACIONES APARECIDAS EN LA COLECCIÓN «AL SERVICIO DE ESPAÑA Y DEL NIÑO ESPAÑOL»
}

\begin{abstract}
Abreviaturas de las bibliotecas, catálogos, repertorios y obras donde se han localizado las monografías:

Biblioteca Nacional (BN)

Biblioteca-Archivo de la Real Academia Nacional de Medicina (RANM)

Biblioteca Nacional de Ciencias de la Salud. (Escuela Nacional de Sanidad) (BNCS)

Catálogo Colectivo de las Bibliotecas Universitarias Españolas (REUNI)

Bosch Marin, J.; Blanco Otero, M.; Mingo, J.M. (1964) Puericultura Social. Madrid, Gráficas González, p. 309-321 (PS)

El lugar de edición sólo se ha consignado cuando era diferente a la ciudad de Madrid. El editor no se ha consignado por ser en todos los casos el mismo, como se ha indicado en el texto: Ministerio de Gobernación, de quien dependía la Dirección General de Sanidad, los Servicios Centrales de Higiene Infantil y la Sección de Maternología, Puericultura e Higiene. El número de páginas se ha indicado cuando aparecía recogido en la fuente consultada.
\end{abstract}

\section{RELACIÓN DE PUBLICACIONES DE LA COLECCIÓN «AL SERVICIO DE ESPAÑA Y DEL NIÑO ESPAÑOL»}

1. MoRALeS, J. L. (1938) La protección de los niños huérfanos, abandonados e indigentes de Sevilla. Valladolid [PS]

2. MoRales, J. L. (1938) Lo que ha hecho Sevilla por los niños desde que se inicio el glorioso Movimiento Nacional. Valladolid [PS-BN]

3. Bosch Marin, J. (1938) Problemas de Maternología y Puericultura. Valladolid. 27 págs. [RANM-PS]

4. IRIZAR, C. (1938) Memoria resumen del servicio de Higiene Infantil de Navarra. Valladolid. 22 págs. [PS]

5. De Prada, J. (1938) Mortalidad infantil en Salamanca. Valladolid. 10 págs. [PS]

6. HERNANDEZ, I. (1938) Los problemas de higiene social infantil en Tenerife. Valladolid. [PS-RANM]

7. NAJERA ANGUlO, L.(1938) Algunos aspectos de la puericultura en España. Valladolid. 19 págs. [PS-RANM]

8. FernANDEZ DE LA PORTILLA, J. (1938) Nociones dermo-venereológicas indispensables a la enfermera puericultora. Valladolid. 19 págs. [PS].

9. MartinEZ VARGaS, A. (1938) Higiene social de la infancia. Valladolid. 25 págs. [PS-BN] 
MODESTA SALAZAR AGULLÓ, EMILIO A. MARTÍNEZ MARCO Y JOSEP BERNABEU-MESTRE

10. AldecoA JuARisti, J. L. (1938) El problema de la lactancia materna durante la dominación roja en Bilbao. Valladolid. 7 págs. [PS]

11. FERRERAS, L. (1938) Lineas generales para un proyecto de educación física en España. Valladolid. 15 págs. [PS-RANM]

12. SUÑER, E. (1938) La alimentación del niño pequeño y su influencia en las cifras de mortalidad. Valladolid. 24 págs. [PS-REUNI]

13. Alvarez Romero, E. (1939) Higiene infantil e instructoras de sanidad. Valladolid. 17 págs. [PS-RANM-BN]

14. Mestre MedinA, J. (1939) La vida de tu nene depende de ti, mujer. Valladolid. [PS-BN]

15. VAllejo De Simon, A. M. (1939) Balance demográfico de un año de guerra. Valladolid, 16 págs. [PS-RANM-BN]

16. Argemi Lloveras, J. (1939) La lucha antituberculosa escolar en Hungría. [PS]

17. Villar Salinas, J. (1939) Distribución por edades de la población de España. 48 págs. [PS-RANM-REUNI]

18. Pedraza CARrasco, L. (1939) Protección del niño antes de su nacimiento. 22 págs. [PS-RANM-BN]

19. Morales, J. L. (1939). Algunas consideraciones acerca de la enfermedad de Feer. 70 págs. [PS-RANM]

20. NAJERA ANGUlO, L. (1939) La natalidad, la mortinatalidad y la mortalidad infantil. 56 págs. [PS-RANM]

21. AldecoA y JUARISTI, J. L. (1939) Antecedentes históricos y aspecto social actual de la lactancia mercenaria. 19 págs. [PS- RANM]

22. Rodriguez Alvarez DE TORNER, M. (1939) La puericultura es a la vez ciencia y arte. 72 págs. [PS-RANM]

23-24. GonZalez Alvarez, M. (1939) Cursillo de iniciación a la puericultura (para madres y futuras madres de la clase obrera). 32 págs. [PS-RANM-REUNI]

25. MARTINEZ VARGAS, A. (1940) Cuidados del niño enfermo. Errores que se cometen en su asistencia. 15 págs. [PS-BN]

26. Rodriguez BABE, R. (1940) Nuevas orientaciones al trabajo social femenino. 27 págs. [PS-RANM]

27. Bosch Marin, J. (1940) Política familiar y sanitaria. 79 págs [PS-RANM]

28. Argemi Lloveras, J. (1940) La vacunación con el B.C.G. en Rumania. [PS-RANM]

29. Villar Salinas, J. (1940) Pasado, presente y futuro de la población de España. 17 págs. [PS-RANM]

30. Navas Migueloa, L.; Pons IBañez, F. (1940) Contribución al estudio médico de los campamentos y colonias escolares. [PS-RANM-BN]

31. Vega FernandeZ, J. (1940) Normas para el estudio constitucional en la infancia. Concepto de los trastornos nutritivos del lactante. 15 págs. [RANM-PS-BN] 
LA SALUD MATERNO-INFANTIL DURANTE EL FRANQUISMO: NOTAS BIBLIOMÉTRICAS...

32. SuAReZ De Puga Reinoso, L. (1940) La enseñanza de la higiene en las escuelas. 26 págs. [RANM-PS]

33. BOIX BARRIOS, J. (1940). Desarrollo del lactante español [RANM-PS]

34. GARRIDO-LeStACHE DiAZ, J. (1940) La infancia y el cinematógrafo. 16 págs. [RANM-PS-BN]

35. COMITE DE REDACCIÓN (1941) Charlas radiofónicas de puericultura. [RANM-PS]

36. RUIZ SANTAMARIA, A. (1941). Ración alimenticia del niño normal. [RANM-PS]

37. COMITE DE REDACCIÓN (1941). Legislación (años 1939-1940 (agotado). [RANM-PS]

38. SELFA, J. (1941). Laboratorio de dietética infantil. [RANM-PS]

39. SOCIAS, A. (1941). El tracoma en la infancia. [RANM-PS-]

40. BoIX BARrios, J. (1941). Diagnostico precoz y sintomatología del Kala-Azar. [RANM-PS-BN]

41. FERNANDEZ Y RUEGAS, D. (1941). La recuperación de los niños lisiados y deformes. [RANM-PS]

42. COMITE DE REDACCIÓN (1941). Ley de sanidad infantil y maternal. [RANM-PS]

43. Rogi Acuña, I. (ViUda De MARTinez Anido) (1941). Preservación de la infancia contra la tuberculosis (obra Brancher). [RANM-PS]

44. COMITE DE REDACCión (1941). Conferencias radiadas de puericultura en Málaga. [RANM-PS]

45. COMITE De REDACCión (1941). Primer semestre de la vida en el niño español. [RANM-PS]

46. COMIte De REDACCión (1941). Normas dietéticas de la escuela nacional de puericultura [RANM-PS]

47. Bosch MARIN, J. (1941). Como ha resuelto la Italia de Mussolini el problema demográfico. 34 págs. [RANM-PS-BN]

48. COMITE De REDACCIÓN (1942) Antecedentes para un plan nacional de obras de puericultura y maternología. 39 págs. [RANM-PS-BN]

49. Aldecoa JuARisti, J. L. (1942) Defensa del niño en los trastornos nutritivos [RANM-PS]

50. Salinas, J. Y SALinAS, P. (1942) Lucha contra la mortalidad infantil en el medio rural [RANM-PS]

51-52. VilLAR SALINAS, J. (1942) La natalidad contemporánea en España. 150 págs. [RANM-PS-BN]

53. VILlAR SALINAS, J. (1942) La escuela nacional de puericultura en el curso de 19411942. 16 págs. [BN-PS]

54. GimeneZ Y GonZALEZ Claramunt, V. (1942) Las mutualidades maternales. [RANM-PS] 
MODESTA SALAZAR AGULLÓ, EMILIO A. MARTÍNEZ MARCO Y JOSEP BERNABEU-MESTRE

55. Blanco Otero, M. (1942) Breves consideraciones sobre la mortalidad infantil. [RANM-PS]

56. Fernandez Perez, J. (1942) Higiene del ejercicio físico y del deporte de los niños de edad escolar. [RANM-PS-BN]

57. BOSCH MARIN, J. (1942) La asistencia sanitaria a la madre y al niño. [RANM-PS]

58. COMITE DE REDACCIÓN (1942) Resumen legislativo del año 1942. [RANM-PS]

59. COMITE DE REDACCIÓN (1942) Programas de sanidad nacional para sus enseñanzas de puericultura, maternologia e higiene escolar. [RANM-PS]

60. MuÑIZ ToCA, F. (1943) La higiene de la boca en el niño. 6 págs. [BN-PS]

61. GIL OsSORIO, F. (1943) Importancia y estudio del medio ambiente del lactante. 24 págs. [RANM-PS-BN]

62. MARTINEZ, M. (1943) Estudio demográfico de un distrito rural. [RANM-PS]

63-64. Bosch Marin, Arce, Ramos, Fernandez, Garrelly De la CAMARA, MuñoYerro Pretel, Blanco Otero, Iturriaga y Bueno (1943) Estudios oficiales primera reunión anual médicos puericultores del estado. 23 págs. [RANM-PS-BN]

65. COMIN, J. (1943) Del folklore medico valenciano. [RANM-PS-BN]

66. MARTINEZ VARGAS, A. (1943) Adherencias prepuciales: su influencia en la precocidad infantil. 18 págs. [RANM-PS]

67. Fernandez Gago Martinez, C. (1943) El trabajo femenino en las distintas profesiones. [RANM-PS]

68. Ros LAVIN, L. (1943) Mortalidad infantil en Zaragoza en el último decenio. [RANMPS]

69. Gonzalez Fernandez, C. (1943) Contribución al estudio de la profilaxis del sarampión por la globulina placentaria y sangre materna. [RANM-PS]

70. GonZalez Del Rio, I. (1943) Tratamiento del raquitismo con dosis masiva única de vitamina D2 y su comparación con el tratamiento clásico. [RANM-PS]

71. LARRAURI GARCIA, M. (1943) Fisiología de la piel del lactante e importancia de su exploración funcional: la reacción cutánea a la histamina. 96 págs. [RANM-PS-BN]

72. SELFA, J. (1944) Paludismo en la infancia. [RANM-PS]

73. No CONSTA AUtORIA (1944) Programas de sanidad nacional. [RANM-PS]

74. SANCHO PASQUAL, V (1944) Memoria de los servicios provinciales de higiene infantil de Castellón. estudio demográfico de la provincia. [RANM-PS]

75. COMITE DE REDACCIÓN (1944) Resumen legislativo de 1943. [RANM-PS]

76. Ruiz SANTAMARIA, A. (1944) Labor dispensarial de higiene infantil. Aspecto sanitario social en Valencia. [RANM-PS-BN-REUNI]

77. AlustiZA IRIARTE, J. (1944) Estudio del niño abandonado en las inclusas. Su mortalidad en España y problemas que plantea. [BN-RANM-PS]

78. COMIN, J. (1944) El problema de las inclusas en España. [RANM-PS] 
79. Alberola Such, R. (1944) Los factores influyentes en la delincuencia infantil. 77 págs. [BN-RANM-PS]

80-81. Arbelo Curbelo, A. (1944) Contribución al estudio del problema de la denatalidad. 27 págs. [RANM-BN-PS]

82. Alonso Muñoyerro, J. A. (1944) La transformación de las inclusas en España: la realidad de la transformación de la de Madrid en Instituto Prov. Puericultura. 86 págs. [RANM-PS-BN-BNCS]

83. Ruiz Santamaria, J. A. (1944) Primera exposición de higiene infantil. 27 págs. [PSRANM].

84-85-86. COMITE DE REDACCiÓN (1945) Servicios de sanidad infantil y maternal (memoria del año 1944). [RANM-PS]

87. SElfa, J. (1945) Memoria del servicio de sanidad infantil de la provincia valencia. 30 págs. [BN-RANM-PS]

88. CONEJO MiR, J. (1945) Memoria resumen de la labor desarrollada por el centro de lucha contra la sifilis congénita de Sevilla, anexo a dichos servicios oficiales. [RANM-BN-PS]

89. ABAd COlOMER; MARTi Alvarez-OSSORIO, I. (1945) Conferencias de maternología $\left(1^{\circ}\right.$ parte $)$. [RANM-PS]

90. Milio Mari Picazo, V.; Alcober Alafont, T. (1945) Conferencias de maternologia ( $2^{a}$ parte). 50 págs. [REUNI-RANM-PS]

91. Clavero Muñoz, A.; Perez Manglano, F.; Valero Estopiña, S.; Marti Pastor, M. (1945) Conferencias de maternología (tercera parte). [RANM-PS]

92. ROYo MONTAÑES, M. (1945) Memoria del servicio de medicina e higiene infantil de Tetuán. 60 págs. [RANM-PS-BN]

93-94. COMITE DE REDACCIÓN (1945) Centros maternales y pediátricos de urgencia de la sanidad nacional. 19 págs. [BN-RANM-PS]

95. Alvarez, P. V. (1945) Contribución al estudio de algunos aspectos epidemiológicos de la infección reumática (en la infancia): distribución de las diversas localizaciones y estudio estadístico comparativo. 18 págs. [RANM]

96. Arbelo Curbelo, A. (1946) La mortalidad infantil en la provincia de Las Palmas capital, provincia sin capital, en el decenio 1928-(1938). 40 págs. [BN-RANM-PS]

97. GIMENEZ, V. (1946) Concepto moderno de un dispensario de higiene infantil. [REUNI-RANM-PS]

98. MARINA BOCANEGRA, G. (1946) Algunas consideraciones generales sobre sifilis congénita precoz, deducidas de su estudio en Málaga. 34 págs. [BN-REUNI-RANM-PS]

99. RuIZ-Zorrilla EnRIQueZ, C. (1946) Estudio de la inmunidad diftérica en Málaga. 28 págs. [BN-RANM-PS]

100-101-102. SELFA, J. (1946) Hospital de niños. 91 págs. [BN-RANM-PS] 
MODESTA SALAZAR AGULLÓ, EMILIO A. MARTÍNEZ MARCO Y JOSEP BERNABEU-MESTRE

103. FRIAS RoIG, A. (1946) Lo que deben saber las madres. 40 págs. [RANM-PS-BN]

104. MILIO MARI, V. (1946) El paludismo en la embarazada y en la puérpera. [RANMBN-PS]

105-106. SANCHO PASQUAL, V. (1946) Crónicas de puericultura regional. [RANM-PS]

107-108. CARDUS, J. (1946) Higiene del embarazo. [RANM-PS-BN]

109-110-111-112. Bosch MARIN, J. (1947) El niño español en el siglo XX. [BN-RANMPS]

113-114. Morcillo, D.; Arbelo Curbelo, A. (1947) Bases para una crítica de la labor dispensarial de higiene infantil. 33 págs. [BN-REUNI-RANM-PS]

115-116. Morales y Gonzalez, J. L. (1947) Morbilidad y mortalidad infantil en Sevilla. 31 págs. [BN- PS-RANM]

117-118. CONTRERAS, F. (1947) Medios eficaces para prevenir la infección hanseniana en la infancia. 43 págs. [BN-PS-RANM]

119-120. Agenjo Cecilia, C. (1947) La leche en la alimentación infantil. 67 págs. [BNPS-RANM]

121. COMITE DE REDACCIÓN (1948) Programas de puericultura, maternología e higiene escolar en 1948. [PS-RANM]

122. Bosch MARIn, J. (1948) Resultados de la vacunación antidiftérica en España durante los últimos tiempos. [RANM-PS]

123-124-125. SELFA, J. (1948) Asistencia hospitalaria infantil en los EEUU: Impresiones de un viaje de estudios. 27 págs. [REUNI-PS-RANM]

126. Garcia Diaz, D. (1948) Plan Provincial de Sanidad infantil y maternal. [BN-PSRANM]

127. BosCh MARIN, J. (1948) La asistencia médico-social en Madrid. [PS-RANM]

128-129. ISERN Y GALCERAN, C. (1948) La mujer en la vida del trabajo. Su misión social, su aspecto jurídico. [BNCS-PS-RANM]

130. Arbelo Curbelo, A. (1948) Asistencia médica a la infancia por pediatraspuericultores de zona. [BNCS-BN-PS-RANM]

131. Turegano, J.; NAVAs MigueloA, L. (1948) Estado actual de la epidemiología y profilaxis de las enfermedades infecciosas en la infancia. 36 págs. [BN-RANM]

132. Loste ECHETO, L. (1949) La imagen del niño en el arte español. 16 págs. [BN-PSRANM]

133-134. Garcia DiAZ, D. (1949) Demografía infantil de la provincia de Soria. [BNCSBN-PS-RANM]

135. Bosch Marin, J.; Blanco Otero, M.; Such SAnchiz, M. (1949) Aportación al VII Congreso Nacional de Pediatría. 19 págs. [BN-PS-RANM]

136-137. CAmacho, M. (1949) Memoria del Centro Maternal de Urgencia de Cieza.15 págs. [BN-PS-BNCS-RANM] 
138. BoIX BARrios, J. (1949) Aspectos sanitarios de un dispensario de puericultura urbano. 71 págs. [RANM-BN-PS-REUNI]

139. AldecoA, IRIZAR y RABADAN, J. L. (1949) Mortalidad por infección en el primer año de la vida. [RANM-BN-PS]

140-141. GiMENEZ, V. (1949) Cursillo de puericultura. 30 págs. [REUNI-RANM-BNPS]

142. Bosch Marin, J.; Blanco Otero, M.; Mingo De Benito, J. M.; Bravo Sanchez DEL PERAL, E. (1949) La puericultura en la sanidad nacional. 47 págs. [RANM-BNPS]

143. PalancA, J. A. (1949) El progreso sanitario de España en la primera mitad del siglo $X X$. [RANM-BN-PS]

144. Arbelo Curbelo, A. (1949) La mortalidad de la infancia en España. [PS-BNRANM]

145. CAÑEllas DOMENECH, F. (1949) Realidad y perspectiva de la maternología. [PSRANM]

146. Bosch Marin, J. (1950) ¿De qué mueren los niños de España? 21 págs. [BNRANM]

147. Comite De Redacción (1950) Bodas de plata del Instituto de Puericultura de Gijón. 15 págs. [PS-BN-BNCS-RANM]

148. Fernanez Turegano, J. (1950) El problema social de la poliomielitis. 15 págs. [PS-RANM-BNCS]

149. Perez Gonzalez, B. (1950) Política sanitaria nacional.[PS-RANM]

150. COMITE DE REDACCiÓN (1950) Cuarta Asamblea de la Hermandad de Enfermeras y asistencia medico-social "Salus Infirmorum". 48 págs. [BN-PS]

151. Loste Echeto, L. (1950) Aspectos deontológicos de la lactancia. 17 págs. [PS]

152. Bosch Marin, J.; De la Camara, J. P.; SAenz De Tejada, V. (1950) 270.000 partos. Sus enseñanzas sanitarias: observaciones sobre la asistencia tocológica prestada en el Seguro Obligatorio de Enfermedad 1947-1949. 45 págs. [RANM-PS-BN]

153-154. BRAVO SANCHEZ DEL PERAL, E. (1950) La sanidad infantil y maternal en España: su organización actual, servicios que comprende, legislación vigente. 22 págs. [RANM-PS-BN]

155. Villar Salinas, J. (1950) Tendencia contemporánea de la mortalidad infantil española. 38 págs. [RANM-BN-PS]

156. CAÑEllas DomeneCh, F. (1951) Significado sanitario-social del dispensario de maternología. [PS-BN]

157. Arbelo Curbelo, A. (1951) La mortalidad neonatal en España (octoenio 194148). 24 págs. [BN-PS]

158. Bosch MARIN, J. (1951) El hospital infantil y maternal en España. 12 págs. [BN-PS] 
159. GonZalez Rodriguez, P. (1951) Epidemia de poliomielitis en España en 1950. 20 págs. [BN-PS-BNCS]

160. BOSCH MARIN, J. (1951) La puericultura del lactante en España. [PS]

161. RAMON, G. (1951) La vacunación contra la difteria por medio de la anatoxina diftérica. [PS-BN]

162. LuQUe BELTRÁN, F. (1951) Deontología del parto sin dolor. [BN-PS-RANM]

163. COMITE DE REDACCIÓN (1951) Programas de puericultura, maternología e higiene escolar en 1951. 64 págs. [BNCS-PS]

164. MORALES, J. L. (1951) La lactancia materna es ineludible deber de conciencia y, desde el punto de vista sanitario, un grave delito su abandono. El papel del medio católico en su defensa. [PS]

165. Loste ECHETO, L.(1951) La defensa de la lactancia materna en España. 16 págs. [PS-BN]

166. Comite De REDACCión (1951) Servicios Centrales de Sanidad Infantil y Maternal (memoria del año 1950). [PS]

167-168. Ruiz SANTAMARIA, J. A. (1952) Estudio evolutivo del garrotillo de los españoles durante la época de Oro de la medicina española. [CSIC-PS-BN-BNCS]

169. COMITE DE REDACCIÓN (1952) Moral y relaciones conyugales. 65 págs. [PS-BNRANM]

170. Garcia Castrillo, L. (1952) El hijo único en Psiquiatría Infantil. 25 págs. [REUNI-PS]

171. Arbelo Curbelo, A. (1952) Necesidad demográfico-sanitaria de rectificar el concepto legal de nacido vivo. [BN-BNCS-PS-RANM]

172. CAÑELLAS DOMENECH, F. (1952) Mortalidad maternal. [BN-PS]

173-174. TORRES MARTY, I. (1952) La protección político-social del niño. [PS]

175. BosCh MARIN, J. (1952) Coloquio de Londres sobre los "niños privados de familia". [BN-PS]

176. COMITE DE REDACCIÓN (1952) Convenio sobre protección de la maternidad: OIT. 15 págs. [BN-PS-RANM]

177. DiReCCION GENERAL DE SANIDAD (1952) Límites morales de los métodos médicos de investigación y tratamiento. 45 págs. [BN-PS]

178. BRAVO SANCHEZ DEL PERAL, E. (1952) La sanidad infantil y maternal en España. [PS-BN]

179. Rodriguez Pedreira, J. (1952) Declaración de la paternidad de los hijos extramatrimoniales. 22 págs. [BNCS-PS-REUNI-BN]

180. Bosch MARIN, J. (1953) Puericultura e higiene mental infantil. [PS]

181-182. SElfa, J. (1953) Reumatismo y corea en la provincia de Valencia. 62 págs. [BN-PS-BNCS] 
183. Mingo DE BENITO, J. (1953) Tutela jurídica del menor. Leyes e instituciones tutelares de menores. [PS]

184. COMITE DE REDACCión (1953) Programas de puericultura, maternología e higiene escolar en 1953. 64 págs. [BN-PS-REUNI]

185. MARINA BocANEGRA, G. (1953) Estudio estadístico-epidemiológico de la leishmaniosis visceral infantil en la provincia de Málaga. [PS-BN]

186-187. COMIN VILLAR, J. (1953) Algunas ideas sobre instalación y sostenimiento de un hospital de niños. [PS-BN]

188-189. LAFFON Y SANCHEZ, J. (1953) El niño en la pintura sevillana. [PS-BN]

190. LOPEZ DE ZUAZO, J. (1953) La organización racional del trabajo escolar en la enseñanza primaria. [PS]

191. BRAVo SANCHEz Del PERAL, E. (1953) Organización y legislación de los servicios de sanidad infantil y maternal en España. [PS-BN-RANM]

192. BosCH MARIN, J. (1954) Salud mental materno-infantil: puericultura y pediatría del alma del niño. 28 págs. [PS-BN-BNCS-]

193. Alcami Garcia, Vazquez Velasco, Tello Matgeli. (1954) Neuropsiquiatría infantil ( $1^{a}$ parte). [PS]

194. MORAGAS, J.; FOLCH. (1954) Neuropsiquiatria infantil (2 ${ }^{a}$ parte) [PS]

195. Arbelo Curbelo, A. (1954) La mortalidad postneonatal en España (1941-1950). [PS-BN-]

196. Blanco Otero, M. (1954) El lactante prematuro. $1^{a}$ parte: asistencia. 23 págs. [PS-BN]

197-198. Arbelo Curbelo, A. (1954) El lactante prematuro. $2^{a}$ parte (concepto, incidencia y mortalidad). 31 págs. [PS-BN-RANM]

199. Bosch MARIN, J. (1954) Enseñanzas del Coloquio Internacional de Amsterdam sobre problemas de salud mental en relación con Sanidad Pública. [PS-BN]

200. Loste Echeto, L. (1954) Fray Tomas de Montalvo, defensor de los expósitos. 20 págs. [PS-BN]

201. Sainz De Los Terreros Amezaga, C.; De Paz Garnelo, J. A. (1954) El Instituto Provincial de Puericultura de Madrid y su morbimortalidad en el decenio 1944-1953. [PS-BNCS]

202. Garcia Martín, M.; SAMANiego Berlanga, J. (1954) La participación española en el Congreso Internacional de Matronas de Londres. 30 págs [PS-RANM]

203. Blanco Otero; Bosch MARIN, J. (1954) UNICEF. [PS-BN]

204-205. COMITE DE REDACCIÓN (1955) Unión Internacional para la educación sanitaria popular. [PS-BN]

206. Comite DE RedAcción (1955) Memoria de los Servicios de Higiene Infantil de Castellón. Estudio demográfico de la provincia. [PS] 
MODESTA SALAZAR AGULLÓ, EMILIO A. MARTÍNEZ MARCO Y JOSEP BERNABEU-MESTRE

207. Fernandez CRehuet, P.; CABrera (1955) Problemas de higiene escolar: (Consideraciones sobre el escolar cardiaco). [PS]

208. DeBRE, R. (1955) Aspecto social de la poliomielitis. 12 págs. [PS-BN-REUNI]

209. Rodriguez EnCINAS, R.; HERnANDEZ, A. (1955) Moral profesional y labor social de la matrona. 60 págs. [PS-BN]

210. DiAZ, C.; GOMEZ, M. (1955) La matrona en el servicio rural. [PS-REUNI]

211. Bueno, J.; Soler SAEZ, J. (1955) La matrona ante los seguros sociales. (Presidentas de los Colegios de Barcelona y Valencia). 21 págs. [PS-BN]

212. Perez Delgado, C. (1955) La morbimortalidad infantil institucional de León. [BNPS]

213. Loste Echeto, L. (1955) Puericultura histórica (algunos casos de lactancia paterna reseñados en libros españoles) [PS]

214. Frias RoIG, A. (1955) Puericultura para hombres. 27 págs. [PS-BN]

215. Bravo SANChez Del Peral, E. (1955) Organización y Legislación de los servicios de sanidad infantil en España. [PS-BNCS]

216. Blanco Otero, M. (1956) Niños, espectáculos públicos y juegos infantiles. 14 págs. [PS-BNCS]

217. BRAVO, E. (1956) Problemas médico-sociales en la infancia. 54 págs. [PS-RANM]

218. COMITE DE REDACCIÓN (1956) El parto sin dolor (método psicoprofiláctico). [PSREUNI]

219. BeCANA CRUZEllas, M. (1956) Morbimortalidad preescolar en Madrid (capital) durante el decenio 1943-1952. 33 págs. [PS-BN]

220. Fernandez CRehuet, P.; CABrera. (1956) Problemas de higiene escolar. [PS-BN]

221-222. COMITE DE REDACCIÓN (1956) El Centro Internacional de la Infancia en España. [PS-BN]

223. BLANCO OTERO, M. (1956) Tres diálogos sobre la salud. 40 págs. [PS-BNCS]

224. Arbelo Curbelo, A. (1956) Evolución y futuro de la mortalidad infantil española. [PS-BN-RANM]

225-226. Sunch SANCHIZ, M. (1956) Acción sanitaria-social sobre la invalidez: (rehabilitación socio-laboral del tuberculoso) 16 págs. [PS-REUNI]

227. SAFFord, F. K.; KURT, J. (1956) Programa Nacional de rehabilitación de niños fisicamente disminuidos. [PS]

228. Bosch Marin, J. (1957) Problemas que plantea actualmente la poliomielitis. 56 págs. [BN-BNCS]

229. TIFON, ZAMORA; FrANDI, F. (1957) Centro auxológico y de medicina preventiva infantil. 28 págs. [PS-]

230. COMITE DE REDACCión (1957) Algunos de los Institutos de Puericultura en España. 87 págs. [PS-BN-BNCS] 
231. ISERN GALCERAN, C. (1957) La salud y la psicología del escolar. 74 págs. [PS-BN]

232. BOIX BARRIOS, J. (1957) La colaboración obstétrico-pediátrica ante el recién nacido. 22 págs. [PS-BN]

233-234. FERnANDEZ De Henestrosa, M. (1957) Información sobre el "social case work". 59 págs [PS]

235. HuERTA NAVES, L. (1957) Algo sobre psicopedagogía infantil. 21 págs. [RANMPS-BN]

236. Torres MARTY, L. (1957) Los problemas médico-sociales del niño invalido. 15 págs. [PS-BN]

237-238. Such SANCHIS, M. (1957) Acción sanitario social sobre la invalidez: un ensayo de rehabilitación en el Sanatorio Nacional de Trillo. 43 págs. [PS-BN]

239. BRAVO, E. (1957) Cuestiones médico-sociales que plantea la organización de un cuerpo de rehabilitación de niños afectos de enfermedad motriz. 36 págs. [PS-BNRANM]

240. COMITE DE REDACCIÓN (1958) Programa de puericultura, maternología e higiene escolar. [PS]

241. BlANCO OTERO, M. (1958) La medicina en la radio. 37 págs. [PS-BN]

242-243. Bosch MARIN, J. Et al (1958) El hospital infantil como centro sanitario. [PSBN-RANM]

244. Blanco Otero, M. (1958) Higiene mental infantil y centros de "child guidance". 30 págs. [PS-BN]

245-246. GIMENEZ, V. (1958) Organización medico-social de un departamento francés. [RANM-PS-BN]

247. SELFA, J. (1958) Memoria del centro de prematuros de Valencia. [PS-BN]

248-249. COMITE DE REDACCiÓN (1958) Aportación española al V Symposium Europeo sobre Poliomielitis. 23 págs. [PS-BN]

250. Bosch MARIN, J. (1958) Encuesta sobre niños fisicamente disminuidos en España. 15 págs. [PS-BN]

251. Arbelo CuRBELo, A. (1958) La mortalidad de la edad preescolar en España. [PS-BN]

252. COMITE DE REDACCIÓN (1959) UNICEF en España. [PS]

253-254. CORTES DE LOS REYES, L. (1959) Los comienzos de la puericultura científica en España. [PS-BN]

255. Arbelo Curbelo, A. (1959) Los niños no deben morir. [PS-REUNI]

256. Blanco Otero, M.; Bravo, E. (1959) Unión Internacional para la Educación sanitaria popular. IV Conferencia Dusseldorf 1959. 45 págs. [PS]

257-258-259-260.COMITE DE REDACCIÓN (1959) Congreso Internacional de matronas. [PS-BN]

261. ISERN YGALCERAN, C. (1960) Los padres ante la educación del niño. [PS-RANM] 
262. Bosch MARIN, J. (1960) Las necesidades de la infancia y de la juventud. [PS-BN]

263-264. Bosch MARIN, J. (1960) Aportación de los organismos internacionales y nacionales a favor de la infancia privada de ambiente familiar normal. [PS-RANM]

265. BOSCH MARIN, J. (1960) El niño y su porvenir profesional. [PS]

266-267. Pertejo de Alcami, J.; Blanco Otero, M. (1960) Bases de la salud mental del niño: periodo comprendido entre el nacimiento y los seis años. [PS]

268. BlANCO OTERO, M. (1960) El niño en la sociedad actual. 41 págs. [PS-BN-BNCS]

269-270. No CONSTA AUTOR (1960) El ambiente familiar y sanitario del niño. [PS-BNREUNI]

271. BERMUdeZ PAREJA, M. (1960) UNICEF: protector de la infancia. 23 págs. [BN-PSBNCS]

272-273. LOSADA, V.; Rodriguez Alvarez DE TORNER, M. (1960) La mujer y la pediatría. 20 págs. [BN-PS-BNCS]

274. BlanCo Soler, C. (1960) La pediatría española y sus maestros. 16 págs. [BN-PSBNCS]

275-276. COMITE De REDACCiÓn (1960) Memoria de la labor desarrollada por los Servicios de Sanidad infantil y maternal durante el año 1960. [PS-RANM]

277. DuOCASTElla, R. (1961) La aportación del servicio social en la atención a la infancia y la adolescencia. 15 págs. [BN-PS]

278-279. Paya Ibars, M.R.; Fdez. de Henestrosa, M.; Pedret, M; Werner, C. (1961) El servicio social y la inadaptación de la infancia y de la juventud. 55 págs. [BN-PS]

280. JUBANY I ARNAU, N. (1961) Los servicios sociales y la prevención contra la delincuencia y la inadaptación social de los menores. [BN-PS]

281-282. CUERDO, B. (1961) La asistencia social y el servicio psiquiátrico infantil. 15 págs. [BN-PS]

283. BuRgos, E. (1961) Reanimación del recién nacido. 46 págs. [BN-PS]

284. TURner, C. E. (1961) Preparación del maestro en educación para la salud. 57 págs. [BN-PS-RANM]

285-286. BOSCH MARIN, J. (1961) Los servicios de sanidad maternal e infantil en Europa: importancia y estudio del medio ambiente del lactante. 15 págs. [BN-PS]

287-288. COMITE DE REDACCIÓN (1962) Memoria de la labor desarrollada por los servicios de sanidad infantil y maternal durante el año 1961. [PS]

289. CoMite De RedACCión (1962) Personal materno-infantil en la sanidad nacional española. [BN-PS]

290. Ruiz Garcia, H. (1962) Aspecto médico, social y laboral de la rehabilitación. 19 págs. [PS]

291-292. FRIAS Y ROIG, A. (1962) Errónea interpretación de la puericultura y verdadero significado de la misma. 25 págs. [BN-PS] 
293. BlanCo Otero, M. (1962) Guarderías infantiles y escuelas maternales. 26 págs. [BN-PS-REUNI-BNCS]

294-295. GUTIERREZ VALDEON, J. (1962) Protección jurídica del menor en la legislación española. 23 págs. [BN-PS]

296-297. PIQUER I JOVER, J. J.; POMES I COLL, C. (1962) Problemas cruciales de la infancia abandonada. 12 págs. [BN-PS-RANM-BNCS]

298. COMITE DE REDACCIÓN (1962) Salud y educación sanitaria. [REUNI-RANM-PS]

299-300. Bosch Marin, J. (1962) Necesidades biológicas de la infancia. 62 págs. [BNPS-REUNI]

301. COMITE DE REDACCIÓN (1963) Veinticinco años de acción sanitaria puericultora. [PS]

302-303. Bosch MARIN et al (1963) Encuesta médico-social sobre el porvenir de los niños con disminuciones fisicas. [PS]

304. SARRION, I (1963) El pediatra ante el problema de los deberes escolares. [RANM- PS]

305-306. Servicios Proteccion Maternal e Infantil (1963) Censo-fichero de centros y personal de los servicios de Sanidad materno-infantil en España. 59 págs. [BNCS-BN]

307. No se ha localizado.

308-309. Ruiz Santamaria, J. A. (1963) Coloquios sobre la salud del escolar: $2^{\circ}$ curso organizado por el Centro Internacional de la Infancia de Paris. 121 págs. [BN]

310. No se ha localizado

311. Serigo SegarRa, A. (1964) Evolución de la mortalidad infantil en España: Bases actuales para la lucha. 91 págs. [BN]

Fecha de recepción: 2 de mayo de 2006

Fecha de aceptación: 8 de octubre de 2006 
